from 71 infants (esophageal atresia (20), duodenal atresia (16), jejunal atresia (7), ileal atresia (11) and anal atresia (17) compared between infants with atresia of upper GIT (AUGIT; esophagus, duodenum) and atresia of lower GIT (ALGIT; jejunum, ileum, colon and anal atresia).

Results There is a significant difference in BW between UGIA and LGIA: BW below the $10^{\text {th }}$ percentile was observed in 16 of 36 patients (44\%) with UGIA and only 6 of 35 patients (17\%) with LGIA ( $p=0.012$, UGIA vs. LGIA). There is no statistical significant difference for BL between both groups ( $p=0.735$ ).

Conclusion Newborn infants with UGIA had lower birth weight than newborn infants with LGIA. This would be in agreement with the hypothesis that absence or decrease of absorption of amniotic fluid in the fetus leads to lower birth weight.

\section{PROSPECTS OF PROBIOTIC BACTERIAL LECTINS IN MEDICINE}

doi:10.1136/archdischild-2012-302724.1404

M Lakhtin, V Lakhtin, S Afanasiev, V Aleshkin, A Aleshkin. Medical Biotechnology, G.N. Gabrichevsky Research Institute for Epidemiology \& Microbiology, Moscow, Russia

Background Probiotic bacterial lectins (PBL) isolated by us from industrial strains of human origin are important regulators of microbiocenoses.

The Aim is to evaluate potential of PBL in medicine.

Results The data obtained indicate that preparations of PBL imitate general functions of probiotics, increase or create new useful activities. In biotopes PBL act as intrinsic tool of microbial selection, factor of supporting probiotic compartment. PBL can be used for screening probiotic microbes, sensitive and resistant clinical strains, and also for monitoring balance of biocenosis in directions "Microbes - Microbes" and "Microbes - Organism". Application of PBL (combinations of L of lactobacilli and bifidobacteria [LL and LB] as the model functional imitator of biotope probiotic compartment) is multidirected and use antipathogen cascade multisynergism of PBL in time and in space. Results indicate that PBL are able to destruct biofilms of pathogen combinations "Staphylococcus > or < Candida" (synergistic contribution: $\mathrm{LL}>$ or $<\mathrm{LB}$ ). LL+LB possess advantages of probiotic action in cases of biotopes where bifidobacteria or lactobacilli are currently absent. It seems, being delivered into organism, PBL will complete cell system "Lactobacillus + Bifidobacterium + Other probiotic-like microbes" and increase synbiotic biotope ingredients. The data on synergistic action between PBL and antibiotics allow using $\mathrm{PBL}$ in cases of non-effectiveness of antibiotics or for decreasing antibiotic doses.

Conclusions Results indicate prospects of PBL in supporting probiotic and synbiotic systems of organism for example during chemotherapy and radiotherapy of tumors, or as ingredients of anti-infectives against eukaryotic pathogens (microfungi and protozoans)

\section{SIGNIFICANT WEIGHT LOSS IN BREASTFED INFANTS IN THE EARLY POSTNATAL LIFE}

doi:10.1136/archdischild-2012-302724.1405

A Mitra, E Parish, AY Sohn, R Chetan. Paediatrics, Southend University Hospitals NHS Foundation Trust, Westcliff-on-Sea, UK

Background and Aims Breastfed newborn infants can develop significant weight loss in early postnatal period associated with hypernatremia. In our hospital, breastfeeding support is provided to mothers both in hospital and community. This breastfeeding care pathway is based on the UNICEF breastfeeding policy. In spite of this sontinuous effort, several newborn infants with significant weight loss need hospital re-admission in the early postnatal period.
Methods A retrospective audit was performed over 6 months (Jan 2011- June 2011) period. Management of all breastfed infants admitted with weight loss (>7\% of birth weight) with or without other clinical symptoms of dehydration were reviewed. Information was collected from infant clinical notes, discharge summaries, hospital pathology reporting system and maternal notes.

Results 1908 infants were born during this period, 33 infants $(1.73 \%)$ required readmissions. No risk factor was identified in 3 infants, 30 infants had one or more risk factors, commonest being caesarean section delivery (61\%) followed by infants born to 'first time mother's (33\%). $72 \%$ of the infants developed jaundice. Majority of the infants $(59 \%)$ were managed with breast feeding support only whereas $15 \%$ needed intravenous fluid support. Most infants (69.6\%) were discharged within 48 hrs of admission. Half of the infants developing hypernatremia had comparatively mild weight loss ( $\leq 10 \%$ of birth weight).

Conclusions Weight loss and Hypernatremia continues to be a problem in neonates particularly in 'at risk' infants. Most of these infants responded well to enteral feeding only. Degree of hypernatremia was not always proportion to the degree of weight loss.

\section{EFFECT OF STORAGE CONTAINER ON BACTERICIDAL} ACTIVITY OF HUMAN MILK

doi:10.1136/archdischild-2012-302724.1406

${ }^{1} S$ Takci, ${ }^{2} \mathrm{D}$ Gulmez, 'S Yigit, ${ }^{2} \mathrm{O}$ Dogan, ${ }^{2} \mathrm{G}$ Hascelik. ${ }^{1}$ Hacettepe University Ihsan Dogramaci Childrens' Hospital; '2Hacettepe University School of Medicine, Department of Clinical Microbiology, Ankara, Turkey

Aim Storage of human milk in refrigerator has been recommended for short term storage. It has been shown that some nutritional, immunological, bioactive properties and bactericidal activity of human milk can alter by refrigeration. Pyrex bottles and polyethylene bags are the two common containers in the setting of storage of human milk. The aim of this study is to compare the effect of the type of storage container on bactericidal activity of human milk at different duration of refrigeration.

Methods Forty four samples of human milk were collected from 22 lactating mother. Two samples of human milk were obtained by manual expression from each mother. One was collected directly into sterile pyrex bottles and the other into polyethylene bags. Each sample was divided into three aliquots, one was processed immediately and remaining two were kept at $4^{\circ} \mathrm{C}$ for 24 and 48 hours. Bactericidal activity of each sample was studied. A strain of E.coli ATCC 25922 was used to determine the bacteridal effect of human milk.

Results Bactericidal activity was significantly reduced in milk samples kept in polyethylene bags compared to the samples kept in pyrex bottles when milk samples stored at $4^{\circ} \mathrm{C}$ for 24 and 48 hours $(\mathrm{p}<0.05)$.

Conclusion Short term storage of human milk in pyrex bottles is more appropriate than polyethylene bags for prevention of decrease in bactericidal activity.

\section{CONTINUOUS GLUCOSE MONITORING IN TERM AND NEAR- TERM INFANTS AT RISK OF HYPOGLYCAEMIA - A PILOT STUDY}

doi:10.1136/archdischild-2012-302724.1407

${ }^{1,2}{ }^{2} \mathrm{D}$ Wackernagel, ${ }^{2} \mathrm{M}$ Dube, 'M Blennow, 2,3y Tindberg. 'Astrid Lindgen's Children Hospital, Karolinska University and Karolinska Institutet, Stockholm; '2Department of Paediatrics, Mälarsjukhuset, Eskilstuna; 'Department of Women's and Children's Health, Uppsala University, Uppsala, Sweden

Background and Aims Postnatal hypoglycaemia is known to increase the risk of adverse neurological outcome in infants at risk. 